\title{
A Computer Program to Simulate NMR Multiplets
}

\author{
Summary \\ Program Mltiplet for Windows calculates first order nuclear magnetic resonance \\ multiplets for spin systems consisting of spin-half nuclei, and draws them on a PC \\ screen. It is offered free of charge.
}

Subject area: Chemistry

\section{Description}

Mltiplet for Windows is a new computer program which we offer free of charge for teaching NMR Spectroscopy.

\section{Type of activity}

A conversational DOS version of Mltiplet was written as an easily used simulation tool, useful in learning to interpret Nuclear Magnetic Resonance spectra of molecules in solution. After over ten years of use, this has now been translated into a full Windows application, with a 'feel' more familiar for today's students. Both the new Windows version and the old DOS version are being offered for download.

\section{Content covered}

Starting from numbers of nuclei and NMR coupling constants in a molecule, Mltiplet draws the expected multiplet, which the chemist can compare with what has been observed using the NMR spectrometer.

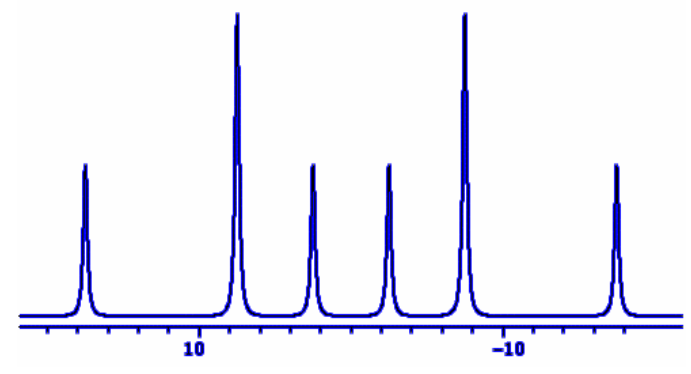

It can be used to simulate the simplest multiplets, but it can equally show the most complex first-order multiplets interpreted in chemical research. While the program generates quite realistic lineshapes, it assumes that the multiplet is completely first order, with intensities of peaks given by Pascal's triangle of binomial coefficients. This is as universally taught in introductory courses on NMR.

\section{Application}

Mltiplet was devised to be used in a drylab environment. Students learn to use tools, such as computer programs, which are the same or similar to those used in research, for working on research-relevant problems in a supervised co-operative group environment. At the introductory level, students can use Mltiplet to find out what happens to the appearance of a multiplet as coupling constants are varied, and to understand the meaning of terms like 'doublet of triplets'. In more advanced courses, 
they can use it to confirm their hand analysis of presented real spectra, thus adding an element of experiment into what would otherwise be a once-through procedure with numerical results. They learn, by trial and error, how to see complex multiplets, and extract coupling constants from them.

\section{Further comments}

While Mltiplet was written as a learning tool for university undergraduates, and has been used mainly at first and second year levels of our honours chemistry courses, it finds occasional use in synthetic chemistry research. We now propose that it can be used also at the secondary school sixth form level, at the very beginning of teaching and learning about NMR. In contrast to the several full simulation and spectrum fitting programs available, in some cases at considerable cost, Mltiplet is not only available free of charge, but it is also extremely easy to download, install, and use. It is envisaged that students may wish to install it in their own PCs, as well as using it in the classroom.

Full notes on use and installation are given on the website below. Detailed instructions and several examples are compiled into the Help facility of the Windows version of the program.

\section{Author(s)}

Dr. Bruce W. Tattershall

Chemistry in the School of Natural Sciences

University of Newcastle upon Tyne

\section{Contact details}

Dr. B.W. Tattershall

Chemistry in the School of Natural Sciences

Bedson Building

University of Newcastle

Newcastle upon Tyne

NE1 7RU

Email: Bruce.Tattershall@ncl.ac.uk

URL

http://www.staff.ncl.ac.uk/bruce.tattershall/software/ 